\title{
Nile Red Staining for Oil Determination in Microalgal Cells: A New Insight through Statistical Modelling
}

\author{
Ronald Halim ${ }^{1}$ and Paul A. Webley ${ }^{2}$ \\ ${ }^{1}$ Bio-Engineering Laboratory (BEL), Department of Chemical Engineering, Monash University, Clayton, VIC 3800, Australia \\ ${ }^{2}$ Department of Chemical and Biomolecular Engineering, The University of Melbourne, Parkville, VIC 3010, Australia \\ Correspondence should be addressed to Ronald Halim; ronaldhalim200486@gmail.com
}

Received 13 June 2015; Revised 18 October 2015; Accepted 9 November 2015

Academic Editor: Doraiswami Ramkrishna

Copyright (c) 2015 R. Halim and P. A. Webley. This is an open access article distributed under the Creative Commons Attribution License, which permits unrestricted use, distribution, and reproduction in any medium, provided the original work is properly cited.

In the wake of global warming and rapid fossil fuel depletion, microalgae emerge as promising feedstocks for sustainable biofuel production. Nile red staining acts as a rapid diagnostic tool to measure the amount of biodiesel-convertible lipid that the cells accumulate. There is a need for the development of a more uniform staining procedure. In its first phase, this study examined the dependence of microalgal Nile red fluorescence (Tetraselmis suecica) in terms of its most pertinent staining variables. A quadratic surface model that successfully described the Nile red fluorescence intensity as a composite function of its variables was generated $\left(r^{2}=0.86\right)$. Cell concentration was shown to have a significant effect on the fluorescence intensity. Up to a certain threshold, fluorescence intensity was shown to increase with Nile red dye concentration. In its second phase, the study reviewed findings from previous Nile red studies to elucidate some of the fundamental mechanism underlying the diffusion of Nile red dye molecules into the microalgal cells and their subsequent interaction with intracellular lipids. Through the review process, we were able to develop a simple framework that provided a set of guidelines for the standardization of the Nile red staining procedure across different microalgal species.

\section{Introduction}

The rapid depletion of fossil fuels together with the effect of greenhouse gas emissions on global climate change has led to an increased commercial interest in biodiesel. Fatty acid methyl ester (FAME) or biodiesel is commercially produced via alkaline-catalyzed transesterification of lipids with methanol [1,2]. Microalgae have been recognized as promising feedstocks for biodiesel as they have the ability to grow rapidly and accumulate large amount of lipid (20$50 \%$ of dry weight). Since many microalgal strains grow in seawater and can be cultivated on nonarable land, large-scale microalgal production should not diminish environmental resources $[1,3-5]$.

There are, however, various technological and economic obstacles that need to be overcome before large-scale production of microalgal biodiesel can take place. The identification of lipid-rich microalgal species and the optimization of cultivation conditions that lead to maximal lipid content are of critical importance [6,7]. A simple method for rapid quantification of lipids in microalgal cells is thus needed to facilitate species identification and cultivation optimization.

The traditional method used for microalgal lipid determination involves organic solvent extraction of the intracellular lipid and subsequent gravimetric measurement of the extracted lipid [1]. To analyze the different lipid fractions, the extracted lipid is then subjected to additional processing steps, such as transesterification and chromatographic separation. This traditional method requires not only timeconsuming and labour-intensive manipulations but also a substantial quantity of biomass. In addition, lipid yield obtained from gravimetric measurement is often underreported as the harsh condition used during the lipid extraction procedure can potentially lead to oxidative decomposition of specific lipid components $[6,8]$.

Nile red staining has frequently been applied as an alternative method for microalgal lipid determination due to its rapidity, simplicity, and low requirement of biomass 
[8]. Nile red is a lipophilic dye whose spectral properties are determined by the polarity of its environment. It emits intense fluorescence in hydrophobic organic solvents and in contact with lipid bodies. On the other hand, in aqueous solutions, its fluorescence is completely quenched. These fluorescence properties made Nile red a natural candidate for lipid staining and quantification $[6,9,10]$.

Even though Nile red staining has been successfully applied for lipid quantification on a number of different microalgal species belonging to Bacillariophyceae and Chlorophyceae classes, the variables that affect the fluorescence intensity have not been investigated at a quantitative level and no kinetic model is yet to be developed [6-9, 11-16]. A mathematical model capable of describing the Nile red fluorescence as a function of different process variables (such as dye concentration, staining duration, and temperature) is needed to identify significant variables and to provide insight into the mechanism underlying the lipid/dye interaction.

Factorial experimental design is a powerful tool to determine optimal experimental conditions. Unlike a traditional experimental strategy whereby only one independent variable is changed from one experiment to another, factorial experimental design allows for multiple independent variables to be simultaneously modified from one experiment to another and takes into account all possible combinations of the assigned values of the independent variables. Such a design evaluates the direct and interactive effects of the independent variables on the response variable $[17,18]$. Response surface analysis on the factorial experimental design enables the development of a regression polynomial model that describes the response variable as a composite function of the independent variables $[17,19]$.

Previous microalgal studies investigating the use of Nile red staining for microalgal application have customized their staining procedures according to their individual purpose [7-9, 11-16], leading to significant difficulties in applying their procedures and comparing their results to other species. Some of these modifications (such as the use of glycerol as a cosolvent) are, however, necessitated in order to improve the dye permeation through the thick and rigid cell walls of certain microalgal species [7]. Because of these divergent literatures, there is currently a need for the establishment of an operating framework that will provide simple guidelines on the optimization of Nile red staining for the various microalgal species. The framework will rule out arbitrary decisions when selecting staining variables and improve the robustness and reliability of the staining method.

This study had dual objectives. The first one was to create a mathematical model that described microalgal Nile red fluorescence as a function of variables of the staining procedure (cell concentration, ultrasonication power, incubation temperature, and Nile red dye concentration). The model would enable the identification of significant variables and elucidate some of the fundamental mechanisms governing lipid/dye interaction. To generate the model, response surface methodology was applied on the results of factorial experiments. The second objective of the study was to develop a simple set of guidelines for the optimization of Nile red staining across various microalgal species. The guidelines would improve the robustness of the staining method across the different species and facilitate a more reliable diagnostic tool for the quantification of biodiesel-convertible lipid in microalgal cells. To create the standardized guidelines, we reviewed findings from previous studies on microalgal Nile red staining and unified them with the insights revealed by our modelling exercise.

\section{Materials and Methods}

2.1. Strain and Cultivation. The microalgal strain used in this study, Tetraselmis suecica (TS), was cultivated in an outdoor bag photobioreactor. The species survived well in outdoor conditions and has previously been shown to have high lipid contents [20]. A modified F growth medium (or MF growth medium) was used for microalgal cultivation. The medium was composed of $150.0 \mathrm{mg} / \mathrm{L} \mathrm{NaNO}_{3}, 22.7 \mathrm{mg} / \mathrm{L} \mathrm{Na}_{2} \mathrm{SiO}_{3} \cdot 5 \mathrm{H}_{2} \mathrm{O}$, $11.3 \mathrm{mg} / \mathrm{L} \mathrm{NaH} \mathrm{PO}_{4} \cdot 2 \mathrm{H}_{2} \mathrm{O}, 9.0 \mathrm{mg} / \mathrm{L} \mathrm{C}_{6} \mathrm{H}_{8} \mathrm{O}_{7} \cdot x \mathrm{Fe}, 9.0 \mathrm{mg} / \mathrm{L}$ $\mathrm{C}_{6} \mathrm{H}_{8} \mathrm{O}_{7}, 0.36 \mathrm{mg} / \mathrm{L} \mathrm{MnCl}_{2} \cdot 4 \mathrm{H}_{2} \mathrm{O}, 0.04 \mathrm{mg} / \mathrm{L} \mathrm{ZnSO} \cdot 7 \mathrm{H}_{2} \mathrm{O}$, $0.02 \mathrm{mg} / \mathrm{L} \mathrm{CoCl} \cdot 6 \mathrm{H}_{2} \mathrm{O}, 0.02 \mathrm{mg} / \mathrm{L} \mathrm{CuSO}_{4} \cdot 5 \mathrm{H}_{2} \mathrm{O}, 0.01 \mathrm{mg} / \mathrm{L}$ $\mathrm{Na}_{2} \mathrm{MoO}_{4} \cdot 2 \mathrm{H}_{2} \mathrm{O}$, trace vitamin $\mathrm{B}_{12}$, biotin, and thiamine dissolved in synthetic seawater. The photobioreactor held approximately $100 \mathrm{~L}$ of microalgal culture and was aerated with compressed air. Cultivation temperature and illumination intensity were dependent on weather conditions. During Melbourne autumn when the cultivation took place, the temperature ranged from 18 to $25^{\circ} \mathrm{C}$ at day time and $12-17^{\circ} \mathrm{C}$ at night time.

2.2. Chemicals and Reagents. Nile red dye (9-(diethylamino)benzo[a]phenoxazin-5 $(5 \mathrm{H})$-one) was purchased from Sigma-Aldrich Pty. Ltd. (Australia). Organic solvents (acetone, isopropanol, and n-hexane) were obtained from commercial suppliers. Nile red dye $(0.01 \mathrm{~g})$ was dissolved in $40 \mathrm{~mL}$ of acetone to produce a Nile red stock solution $(0.25 \mathrm{mg} / \mathrm{mL})$.

2.3. Preliminary Nile Red Staining and Lipid Extraction. Microalgal culture (a total of $2.1 \mathrm{~L}$ for all experiments in Sections 2.3 and 2.4) was sampled from the photobioreactor. Nile red stock solution $(0.04 \mathrm{~mL})$ was added to $4 \mathrm{~mL}$ of the collected microalgal culture in a clear-sided plastic cuvette. The cuvette was inverted several times to ensure thorough mixing. To analyze the development of fluorescence with poststaining time, the fluorescence intensity of the stained mixture was recorded at regular intervals with a fluorescence spectrophotometer (RF-1501, Shimadzu Corporation, Japan) for $30 \mathrm{~min}$ at excitation and emission wavelengths of 530 and $580 \mathrm{~nm}$, respectively [7].

A portion of the microalgal culture was dewatered using a bench-top centrifuge (Heraeus Multifuge 3 S-R, Kendro, Germany) at $4500 \mathrm{rpm}$ for $10 \mathrm{~min}$. The supernatant was discarded and the microalgal paste was rinsed with deionised water to remove residual salts. The resuspended microalgal culture was recentrifuged and the resulting microalgal paste was dried at $60^{\circ} \mathrm{C}$ in the oven (Model UNE 500 PA, Memmert $\mathrm{GmbH}+\mathrm{Co}$., Germany) for $16 \mathrm{~h}$. From weighing the dried biomass, microalgal cell concentration of the culture used for the above staining was determined to be $0.11 \pm 0.005 \mathrm{~g}$ dried 
TABLE 1: Real and coded values of the independent variables investigated in the factorial experimental design.

\begin{tabular}{|c|c|c|c|c|c|}
\hline \multirow{2}{*}{ Microalgal cell concentration } & Real value (g dried microalgae/L culture) & & 0 & 0.11 & \\
\hline & Coded value & & 0 & 1 & \\
\hline \multirow{2}{*}{ Ultrasonication power } & Real value $(\mathrm{W})$ & 0 & 65 & 130 & \\
\hline & Coded value & -1 & 0 & 1 & \\
\hline \multirow{2}{*}{ Incubation temperature } & Real value $\left({ }^{\circ} \mathrm{C}\right)$ & 20 & 30 & 40 & \\
\hline & Coded value & -1 & 0 & 1 & \\
\hline \multirow{2}{*}{ Nile red dye concentration } & Real value ( $\mu$ g dye/mL culture) & 0 & 2.5 & & 6.25 \\
\hline & Coded value & -1 & 0 & & 1.5 \\
\hline
\end{tabular}

microalgae/L feed mixture. A pestle and mortar was then used to grind the dried microalgal biomass into powder.

A Soxhlet apparatus was used for complete lipid extraction from the biomass. Description of a typical Soxhlet operation can be found elsewhere [21]. A cellulose thimble containing the microalgal powder was placed inside the extraction chamber of the Soxhlet apparatus. Isopropanol $(300 \mathrm{~mL})$ was used to extract the microalgal lipid for $2 \mathrm{~h}$ at the rate of 25 refluxes per hour. Once isopropanol was removed from the apparatus, $\mathrm{n}$-hexane $(300 \mathrm{~mL})$ was used to extract the microalgal lipid for $1 \mathrm{~h}$ at the rate of 43 refluxes per hour. Soxhlet extraction achieved complete recovery of a lipid fraction through continuous reestablishment of lipid mass transfer equilibrium between the biomass and the specific solvent. The sequential use of two organic solvents of opposite polarity (isopropanol was highly polar, while nhexane was nonpolar) in our method ensured the complete recovery of both lipid fractions (polar and neutral) and thus all intracellular lipids from the biomass. At the end of the hexane extraction, the microalgal powder in the thimble was completely bleached while the hexane batch in contact with the powder was colourless, both indicators demonstrating that all of the available lipids in the biomass have been completely extracted. Both hexane and isopropanol phases were combined and transferred to preweighed flasks before being evaporated to yield the extracted lipid. The lipid yield was determined gravimetrically.

2.4. Investigation of Nile Red Staining Using a Factorial Experimental Design. A factorial experimental design was used to evaluate the effect of four independent staining variables (cell concentration, ultrasonication power, incubation temperature, and Nile red dye concentration) on Nile red fluorescence. The reasons for the selection of these variables are elaborated in Section 3. Table 1 shows the different values of the independent variables being investigated. With the exception of cell concentration which only had two values $(0$ and $0.11 \mathrm{~g}$ dried microalgae/L feed mixture), all of the other variables were assigned three distinct levels. Ultrasonication on the feed mixture was carried out at three different power levels $(0,65$, or $130 \mathrm{~W})$. The feed mixture was incubated at three separate temperatures $\left(20,30\right.$, or $\left.40^{\circ} \mathrm{C}\right)$, while the amount of Nile red dye added to the feed mixture was varied at the following concentrations: $0,2.5$, or $6.25 \mu \mathrm{g}$ dye/mL culture. A total of forty experiments encompassing the different combinations of the assigned values of the independent variables were performed (Table 2).

2.4.1. Factorial Experiments. The culture collected in Section 2.3 was used for the factorial experiments in this section. To investigate the effect of cell concentration on the Nile red staining efficiency, the experimental design assessed the difference in fluorescence intensity between stained microalgal culture (microalgal cell concentration $=0.11 \mathrm{~g}$ dried microalgae/L culture) and stained MF growth medium (microalgal cell concentration $=0 \mathrm{~g}$ dried microalgae/L culture). The MF growth medium for the staining experiments was derived by filtering a portion of the microalgal culture sampled above $(400 \mathrm{~mL})$ through Whatman number 1 paper $(150 \mathrm{~mm}$ diameter).

To investigate the effect of ultrasonication power on Nile red staining efficiency, the experimental design evaluated the difference in fluorescence intensity between stained mixtures with intensely presonicated feed mixtures (ultrasonication power $=130 \mathrm{~W}$ ), mildly presonicated feed mixtures (ultrasonication power $=65 \mathrm{~W}$ ), or unsonicated feed mixtures (ultrasonication power $=0 \mathrm{~W}$ ). $125 \mathrm{~mL}$ of feed mixture (either microalgal culture or growth medium) was processed with an ultrasonicator (Vibra-Cell Model VCX134PB) manufactured by Sonics \& Materials, Inc. (USA). The probe of the ultrasonicator (6 $\mathrm{mm}$ in diameter and $113 \mathrm{~mm}$ in length) was immersed into the feed mixture to deliver ultrasound at a frequency of $40 \mathrm{kHz}$ and selected acoustic power level $(0,65$, or $130 \mathrm{~W})$ for $20 \mathrm{~min}$.

Out of the $800 \mathrm{~mL}$ of microalgal culture used for the factorial experiments, $400 \mathrm{~mL}$ was used as a feed mixture, while $400 \mathrm{~mL}$ was filtered as mentioned above to produce the MF growth medium feed mixture. The feed mixture (either microalgal culture or growth medium) was divided into three and ultrasonicated according to the above description $(0 \mathrm{~W}$, $65 \mathrm{~W}$, or $130 \mathrm{~W})$. The sonicated feed mixture was pipetted as $4 \mathrm{~mL}$ aliquots into clear-sided cuvettes. Each cuvette was incubated at a select temperature $\left(20,30\right.$, or $\left.40^{\circ} \mathrm{C}\right)$ for 10 minutes in an oven (Model UNE 500 PA, Memmert $\mathrm{GmbH}+\mathrm{Co}$., Germany). The sonicated and incubated feed mixture in the cuvette was then stained with Nile red stock solution. The volume of Nile red stock solution added was appropriately adjusted to produce one of the following Nile red dye concentrations: $0,2.5$, or $6.25 \mu \mathrm{g}$ dye/mL culture. The stained mixture was inverted several times to ensure 
TABLE 2: Factorial experimental design matrix and values of the observed fluorescence intensity (also referred to as experimental results). Fluorescence intensity was determined at excitation wavelength of $530 \mathrm{~nm}$, emission wavelength of $607 \mathrm{~nm}$, and $15 \mathrm{~min}$ poststaining time.

\begin{tabular}{|c|c|c|c|c|c|}
\hline $\begin{array}{l}\text { Experiment } \\
\text { number }\end{array}$ & $\begin{array}{l}\text { Microalgal cell } \\
\text { concentration }\end{array}$ & $\begin{array}{l}\text { Ultrasonication } \\
\text { power }\end{array}$ & $\begin{array}{l}\text { Incubation } \\
\text { temperature }\end{array}$ & $\begin{array}{c}\text { Nile red dye } \\
\text { concentration }\end{array}$ & $\begin{array}{c}\text { Observed fluorescence } \\
\text { intensity (afu) }\end{array}$ \\
\hline 1 & 0 & -1 & -1 & -1 & - \\
\hline 2 & 0 & -1 & -1 & 0 & 62.07 \\
\hline 3 & 0 & -1 & -1 & 1.5 & 72.41 \\
\hline 4 & 0 & -1 & 0 & -1 & - \\
\hline 5 & 0 & -1 & 0 & 0 & 63.52 \\
\hline 6 & 0 & -1 & 0 & 1.5 & 90.99 \\
\hline 7 & 0 & -1 & 1 & -1 & - \\
\hline 8 & 0 & -1 & 1 & 0 & 90.60 \\
\hline 9 & 0 & -1 & 1 & 1.5 & 152.14 \\
\hline 10 & 0 & 0 & -1 & -1 & - \\
\hline 11 & 0 & 0 & -1 & 0 & 44.83 \\
\hline 12 & 0 & 0 & -1 & 1.5 & 70.69 \\
\hline 13 & 0 & 0 & 0 & -1 & - \\
\hline 14 & 0 & 0 & 0 & 0 & 70.69 \\
\hline 15 & 0 & 0 & 0 & 1.5 & 100.00 \\
\hline 16 & 0 & 0 & 1 & -1 & - \\
\hline 17 & 0 & 0 & 1 & 0 & 41.38 \\
\hline 18 & 0 & 0 & 1 & 1.5 & 110.34 \\
\hline 19 & 0 & 1 & -1 & -1 & - \\
\hline 20 & 0 & 1 & -1 & 0 & 51.50 \\
\hline 21 & 0 & 1 & -1 & 1.5 & 72.10 \\
\hline 22 & 0 & 1 & 0 & -1 & - \\
\hline 23 & 0 & 1 & 0 & 0 & 63.79 \\
\hline 24 & 0 & 1 & 0 & 1.5 & 120.69 \\
\hline 25 & 0 & 1 & 1 & -1 & - \\
\hline 26 & 0 & 1 & 1 & 0 & - \\
\hline 27 & 0 & 1 & 1 & 1.5 & - \\
\hline 28 & 1 & -1 & -1 & -1 & 18.88 \\
\hline 29 & 1 & -1 & -1 & 0 & 200.86 \\
\hline 30 & 1 & -1 & -1 & 1.5 & 137.34 \\
\hline 31 & 1 & -1 & 0 & -1 & 8.58 \\
\hline 32 & 1 & -1 & 0 & 0 & 123.61 \\
\hline 33 & 1 & -1 & 0 & 1.5 & 140.77 \\
\hline 34 & 1 & -1 & 1 & -1 & 17.24 \\
\hline 35 & 1 & -1 & 1 & 0 & 177.59 \\
\hline 36 & 1 & -1 & 1 & 1.5 & 153.45 \\
\hline 37 & 1 & 0 & -1 & -1 & 17.24 \\
\hline 38 & 1 & 0 & -1 & 0 & 122.41 \\
\hline 39 & 1 & 0 & -1 & 1.5 & 131.03 \\
\hline 40 & 1 & 0 & 0 & -1 & 15.52 \\
\hline 41 & 1 & 0 & 0 & 0 & 106.90 \\
\hline 42 & 1 & 0 & 0 & 1.5 & 148.28 \\
\hline 43 & 1 & 0 & 1 & -1 & 20.69 \\
\hline 44 & 1 & 0 & 1 & 0 & 148.28 \\
\hline 45 & 1 & 0 & 1 & 1.5 & 158.62 \\
\hline
\end{tabular}


TABLE 2: Continued.

\begin{tabular}{lccccc}
\hline $\begin{array}{l}\text { Experiment } \\
\text { number }\end{array}$ & $\begin{array}{c}\text { Microalgal cell } \\
\text { concentration }\end{array}$ & $\begin{array}{c}\text { Ultrasonication } \\
\text { power }\end{array}$ & $\begin{array}{c}\text { Incubation } \\
\text { temperature }\end{array}$ & $\begin{array}{c}\text { Nile red dye } \\
\text { concentration }\end{array}$ & $\begin{array}{c}\text { Observed fluorescence } \\
\text { intensity (afu) }\end{array}$ \\
\hline 46 & 1 & 1 & -1 & -1 & 17.17 \\
47 & 1 & 1 & -1 & 0 & 78.97 \\
48 & 1 & 1 & -1 & 1.5 & 101.29 \\
49 & 1 & 1 & 0 & -1 & 27.59 \\
50 & 1 & 1 & 0 & 0 & 79.31 \\
51 & 1 & 1 & 0 & 1.5 & 131.03 \\
52 & 1 & 1 & 1 & -1 & - \\
53 & 1 & 1 & 1 & 0 & - \\
\hline
\end{tabular}

complete mixing and was further incubated at its respective temperature in the oven for 15 minutes. Emission spectrum of the stained mixture was recorded with a fluorescence spectrophotometer (RF-1501, Shimadzu Corporation, Japan) at an excitation wavelength of $530 \mathrm{~nm}$. The fluorescence intensity at an emission wavelength of $607 \mathrm{~nm}$ was manually determined from the captured emission spectrum.

2.4.2. Statistical Analysis. Response surface analysis was applied to the results of the experimental design in Section 2.4.1. In order to describe fluorescence intensity as a composite function of the four independent variables in the experimental design, the observed fluorescence intensities were fitted to the quadratic model:

$$
F=\beta_{0}+\sum \beta_{i} X_{i}+\sum \beta_{i i} X_{i}^{2}+\sum \beta_{i j} X_{i} X_{j},
$$

where $F$ is the fluorescence intensity (afu) at excitation wavelength of $530 \mathrm{~nm}$ and emission wavelength of $607 \mathrm{~nm}$; $\beta_{0}$ is the intercept regression coefficient; $\beta_{i}$ is the linear main regression coefficient of the independent variable; $\beta_{i i}$ is the quadratic main regression coefficient of the independent variable; $\beta_{i j}$ is the interaction regression coefficient of the independent variable; $X_{i}$ is the coded value of the independent variable.

In the quadratic model, the values of independent (or input) variables were coded according to the following equation:

$$
X_{i}=\frac{z_{i}-z_{\mathrm{cp}}}{\Delta z_{i}}
$$

where $X_{i}$ is the coded value of the independent variable $i$; $z_{i}$ is the real value of the independent variable $i ; z_{\mathrm{cp}}$ is the real value of the independent variable $i$ at the centre point; $\Delta z_{i}$ is the step change of the real value of the independent variable $i$ corresponding to a single-unit variation of the coded value of the independent variable $i$. For an independent variable that only has two values (cell concentration in our case), $z_{\mathrm{cp}}$ becomes the lower value of the independent variable. Table 1 shows the real values of the independent variables and their corresponding coded values.

The regression coefficients of the model were subjected to $p$ value tests in order to determine the statistical significance of the main and interaction effects of the independent variables, while overall goodness of fit of the model was evaluated using determination coefficient $R^{2}$. 3D response surface plots of fluorescence intensity as predicted by the quadratic model were generated to enable visualization of the complex relationships between fluorescence intensity and the independent variables (in coded values). Statistica Version 9 software package (StatSoft Pacific Pty. Ltd., Australia) was used to carry out all of the statistical works.

2.4.3. Intact Cell Count. The efficiency of ultrasonication in disrupting microalgal cells was evaluated via reduction in the intact cell counts. A small sample of the microalgal culture $(10 \mu \mathrm{L})$ was collected before and after the ultrasonication processes $(0,65$, or $130 \mathrm{~W})$ in Section 2.4.1. Intact cell count was determined with a standard Neubauer haemocytometer under a light microscope (Olympus BX51 equipped with Moticam 1000 camera). For each sample, between 7 and 20 cell counts at different grids in the haemocytometer were carried out. The intact cell counts for samples collected before ultrasonication averaged out at 1,557 \pm 837 cells $/ \mu \mathrm{L}$. The degree of disruption or $D$ (\% of intact cells) for a particular ultrasonication process was calculated according to

$$
D=\frac{C_{b}-C_{a}}{C_{b}} \cdot 100,
$$

where $C_{b}$ is the average intact cell count of the culture before the ultrasonication and $C_{a}$ is the average intact cell count of the culture after the ultrasonication.

\section{Results and Discussion}

3.1. Preliminary Nile Red Staining Results. Preliminary observation under the microscope indicated that the Nile red dye was able to interact with the intracellular lipid globules within the TS cells. As can be seen in the microscopic images (Figure 1), the lipid globules emitted orange/yellow fluorescence upon interaction with the Nile red dye, while chlorophyll emitted a red autofluorescence.

Figure 2 shows the evolution of fluorescence intensity of stained TS culture as a function of poststaining time. The choice of excitation and emission wavelengths $(530 \mathrm{~nm}$ and 


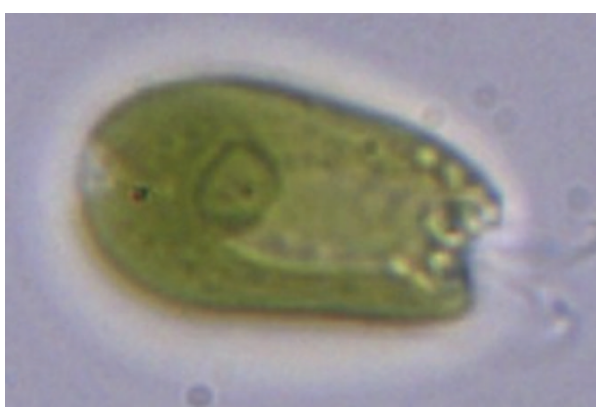

(a)

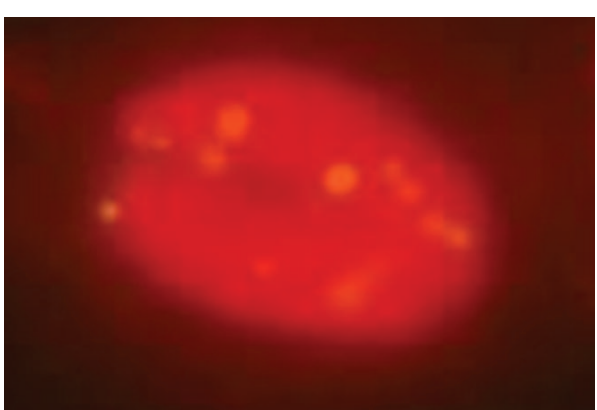

(b)

Figure 1: Microscopic images of a Tetraselmis suecica cell. (a) Cell before being stained by the Nile red dye. (b) Cell after being stained by the Nile red dye.

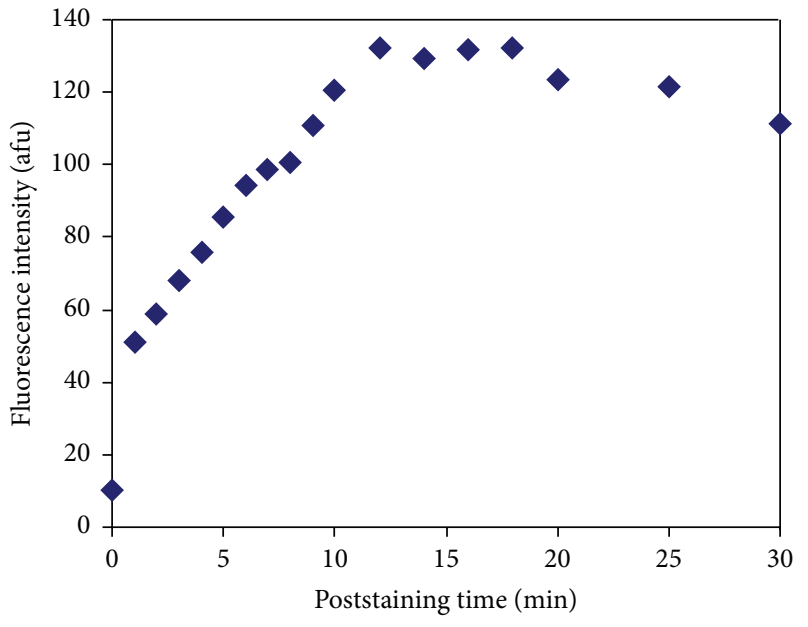

FIGURE 2: The effect of poststaining time on the fluorescence intensity of stained microalgal culture. Excitation wavelength $=$ $530 \mathrm{~nm}$ and emission wavelength $=580 \mathrm{~nm}$.

$580 \mathrm{~nm}$, resp.) was taken from the work of Chen et al. [7] on Chlorella vulgaris, another green microalgal species. In Figure 2, fluorescence intensity appeared to increase rapidly in the first ten minutes before eventually reaching a peak at around $15 \mathrm{~min}$. After this optimal time, the fluorescence intensity experienced a slight decline for the rest of the duration in which the measurement was taken. This optimal poststaining time $(15 \mathrm{~min})$ fell comfortably within the range generally reported for microalgal Nile red staining (Table 4).

Table 4 is a summary of the optimal parametric values reported by previous microalgal Nile red studies. Based on the table, each microalgal species appears to have its own optimal poststaining time. Such a wide diversity is expected as cell wall thickness varies dramatically between species and fluorescence enhancement is known to be a direct function of how quickly the dye can permeate through the cell wall/membrane to interact with the lipid. The reason for fluorescence quenching after optimal poststaining time is yet to be fully understood.

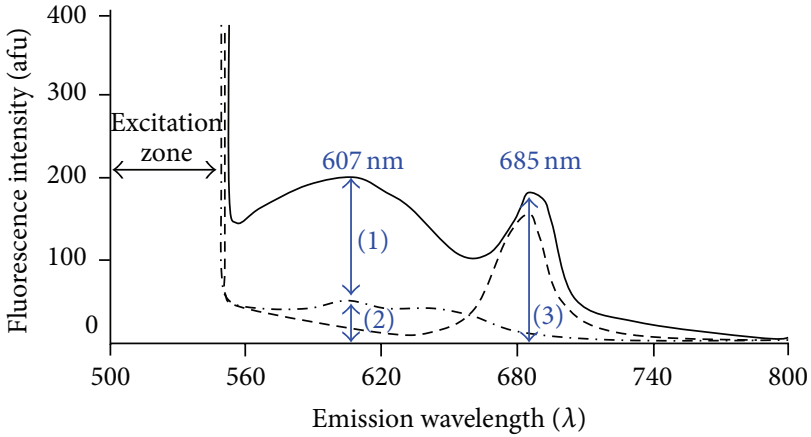

(1) Dye/lipid interaction fluorescence

(2) Dye/medium interaction fluorescence

(3) Chlorophyll autofluorescence

FIGURE 3: Emission spectra of three different experiments from the factorial experimental design (Table 2). Excitation wavelength = $530 \mathrm{~nm}$. For convenience, real values (instead of coded values) of the independent variables are shown below. For all three experiments, ultrasonication power $=0 \mathrm{~W}$, incubation temperature $=20^{\circ} \mathrm{C}$, and poststaining time $=15 \mathrm{~min}$. - : experiment 29 (stained microalgal culture); cell concentration $=0.11 \mathrm{~g}$ dried microalgae $/ \mathrm{L}$ culture and Nile red dye concentration $=2.5 \mu \mathrm{g}$ dye $/ \mathrm{mL}$ culture. $\cdots$. $\because$ experiment 2 (stained growth medium); cell concentration = $0 \mathrm{~g}$ dried microalgae $/ \mathrm{L}$ culture and Nile red dye concentration $=$ $2.5 \mu \mathrm{g}$ dye/mL culture. - - - -: experiment 28 (unstained microalgal culture); cell concentration $=0.11 \mathrm{~g}$ dried microalgae $/ \mathrm{L}$ culture and Nile red dye concentration $=0 \mu \mathrm{g}$ dye $/ \mathrm{mL}$ culture.

\subsection{Factorial Experimental Design Results}

3.2.1. Selection of Optimal Emission Wavelength. Our preliminary Nile red staining indicated that emission wavelength in the range of $605-610 \mathrm{~nm}$ was likely to be a more optimum choice for TS cells than $580 \mathrm{~nm}$ (results not shown). For this reason, we examined the fluorescence emission spectra obtained from the factorial design experiments in Section 2.4. Figure 3 shows the fluorescence emission spectra from experiments 29,2 , and 28 (refer to Table 2 for details). To provide a fair comparison, we have selected experiments with the same conditions for ultrasonication power $(0 \mathrm{~W})$ and 
incubation temperature $\left(20^{\circ} \mathrm{C}\right)$. Experiment 2 is the stained growth medium (microalgal cell concentration $=0 \mathrm{~g}$ dried microalgae/L culture, Nile red dye concentration $=2.5 \mu \mathrm{g}$ dye $/ \mathrm{mL}$ culture). Its emission profile showed a peak at $607 \mathrm{~nm}$ (fluorescence intensity $=62.07 \mathrm{afu}$ ). This peak resulted from the background interaction of the Nile red dye with the salts in the growth medium and had to be taken into consideration when reporting the efficiency of Nile red process in staining microalgal lipids.

Experiment 29 is the stained microalgal culture (microalgal cell concentration $=0.11 \mathrm{~g}$ dried microalgae $/ \mathrm{L}$ culture, Nile red dye concentration $=2.5 \mu \mathrm{g}$ dye $/ \mathrm{mL}$ culture). In its emission profile, the same peak that appeared in experiment 2 at $607 \mathrm{~nm}$ was substantially increased in size (fluorescence intensity $=200.86 \mathrm{afu}$ ) as the Nile red dye was able to interact with both the salts in the growth medium and the lipid in the microalgal cells. Based on this emission profile, $607 \mathrm{~nm}$ appeared to be the optimum emission wavelength for dye/lipid interaction of our microalgal species (TS). This emission wavelength was thus used for all fluorescence measurements in the factorial design.

Experiment 28 is the unstained microalgal culture (microalgal cell concentration $=0.11 \mathrm{~g}$ dried microalgae $/ \mathrm{L}$ culture, Nile red dye concentration $=0 \mu \mathrm{g}$ dye $/ \mathrm{mL}$ culture). Even though its fluorescence emission profile expectedly did not have any of the dye peaks at $607 \mathrm{~nm}$, it showed a later peak at $685 \mathrm{~nm}$. We attributed this peak to the autofluorescence of chlorophyll in the microalgal cells. As expected, the chlorophyll autofluorescence peak was also present in the emission profile of experiment 29 (stained microalgal culture) but was absent in that of experiment 2 (stained growth medium). The results of this fluorometric analysis were consistent with our earlier microscopic observations that showed stained lipid globules to be fluorescing at the orange/yellow colour range (560-635 nm) and chlorophyll to be autofluorescing at the red colour range $(635-700 \mathrm{~nm})$.

Our excitation and emission wavelengths $(530 / 607 \mathrm{~nm})$ fell within the range generally reported to be optimal for microalgal Nile red staining (Table 4). Variations in the optimal values of excitation and emission wavelengths are known to depend on the lipid contents and compositions of the investigated microalgal species (refer to Section 3.3). As a general rule, the emission wavelength of lipid fluorescence increases with lipid polarity $[10,14,15]$. Thus, the emission of polar lipids generally peaks in the orange zone $(590-635 \mathrm{~nm})$, while neutral lipids have optimal emission fluorescence in the yellow zone $(560-590 \mathrm{~nm})[14,15]$.

3.2.2. Statistical Analysis. We now discuss the statistical analysis of the factorial experimental design. Table 1 shows the real and coded values of the independent variables being investigated, while Table 2 lists all of the experiments being carried out under the factorial experimental design matrix together with their observed fluorescence intensities. Based on the above analysis of optimum emission wavelength and poststaining time, fluorescence values of all factorial experiments were captured at $607 \mathrm{~nm}$ and $15 \mathrm{~min}$ after staining. According to the results of lipid extraction in Section 2.3, the microalgal culture was found to have a cell concentration of
TABLE 3: Polynomial modelling of fluorescence intensity as a composite function of the four independent variables. Results (observed fluorescence intensities) of factorial experimental design as displayed in Table 2 were used for the modelling. Fluorescence intensity was recorded at excitation wavelength of $530 \mathrm{~nm}$ and emission wavelength of $607 \mathrm{~nm}$. The regression coefficients and $p$ values of the linear main effects, the quadratic main effects, and the interaction effects of the four independent variables are reported. $X_{1}=$ coded value of cell concentration, $X_{2}=$ coded value of ultrasonication power, $X_{3}=$ coded value of incubation temperature, and $X_{4}=$ coded value of Nile red dye concentration.

\begin{tabular}{lccc}
\hline $\begin{array}{l}\text { Model } \\
\text { term }\end{array}$ & $\begin{array}{c}\text { Regression } \\
\text { coefficient }\end{array}$ & $\begin{array}{c}\text { Standard } \\
\text { error }\end{array}$ & $p$ value \\
\hline Intercept & 55.52 & 10.57 & $\mathrm{n} / \mathrm{a}$ \\
$X_{1}$ & $\mathbf{6 5 . 9 5}$ & $\mathbf{1 1 . 2 5}$ & $\mathbf{3 . 5 2 3} * \mathbf{1 0}^{-\mathbf{6}}$ \\
$X_{2}$ & 1.15 & 8.74 & 0.896 \\
$X_{2}^{2}$ & 5.80 & 7.95 & 0.472 \\
$X_{3}$ & 14.71 & 8.74 & 0.104 \\
$X_{3}^{2}$ & 7.36 & 7.95 & 0.363 \\
$X_{4}$ & $\mathbf{8 9 . 9 7}$ & $\mathbf{1 2 . 2 1}$ & $\mathbf{7 . 9 1 5} * \mathbf{1 0}$ \\
$X_{4}^{2}$ & $-\mathbf{4 2 . 6 2}$ & $\mathbf{6 . 4 4}$ & $\mathbf{5 . 1 2 9} * \mathbf{1 0}$ \\
$X_{1} X_{2}$ & -14.35 & 9.86 & 0.158 \\
$X_{1} X_{3}$ & -7.56 & 9.86 & 0.450 \\
$X_{1} X_{4}$ & -19.76 & 10.45 & 0.070 \\
$X_{2} X_{3}$ & 5.69 & 7.50 & 0.455 \\
$X_{2} X_{4}$ & -0.72 & 4.98 & 0.887 \\
$X_{3} X_{4}$ & 8.37 & 4.98 & 0.105 \\
\hline
\end{tabular}

$0.11 \mathrm{~g}$ dried microalgae/L culture and a total intracellular lipid content of $0.29 \mathrm{~g}$ crude lipid/g dried microalgae.

Table 3 shows the regression coefficients obtained when observed fluorescence intensities in Table 2 were fitted to the quadratic model. The model can be written out as

$$
\begin{aligned}
F= & 55.52+65.95 X_{1}+1.15 X_{2}+5.80 X_{2}^{2}+14.71 X_{3} \\
& +7.36 X_{3}^{2}+89.97 X_{4}-42.62 X_{4}^{2}-14.35 X_{1} X_{2} \\
& -7.56 X_{1} X_{3}-19.76 X_{1} X_{4}+5.69 X_{2} X_{3} \\
& -0.72 X_{2} X_{4}+8.37 X_{3} X_{4}
\end{aligned}
$$

where $F$ is the fluorescence intensity (afu), $X_{1}$ is the coded value of microalgal cell concentration, $X_{2}$ is the coded value of ultrasonication power, $X_{3}$ is the coded value of incubation temperature, and $X_{4}$ is the coded value of Nile red dye concentration.

The statistical significance of each independent variable on fluorescence intensity was determined via $p$ value tests on its linear main, quadratic main, and interaction regression coefficients. The smaller the $p$ value, the more significant the corresponding regression coefficient. $p$ value $<0.01$ indicated the regression coefficient to be statistically significant at $95 \%$ confidence level. As can be seen from Table 3, the regression coefficients that achieved statistical significance were (in descending order) the linear main term of Nile red dye concentration $\left(p\right.$ value $=7.915 * 10^{-8}$ ), the quadratic main 


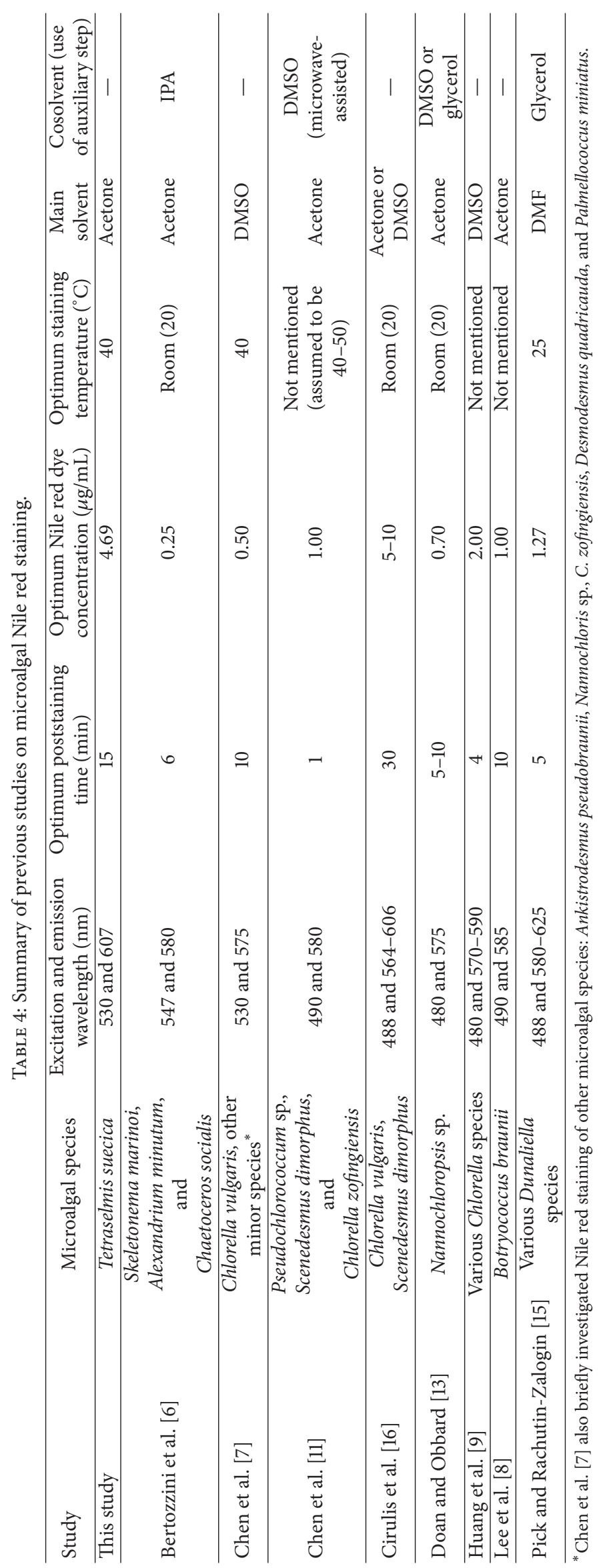




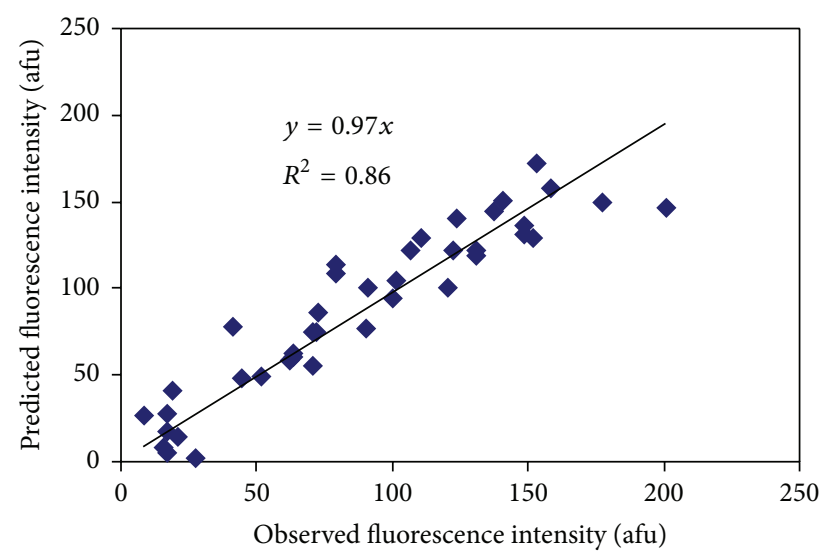

FIGURE 4: Fluorescence intensity predicted by the quadratic model versus fluorescence intensity observed in the experiments. Refer to Table 2 for experimental conditions and observed fluorescence intensity values. Refer to Table 3 for regression coefficients of the quadratic models. Fluorescence intensity was determined at excitation wavelength of $530 \mathrm{~nm}$ and emission wavelength of $607 \mathrm{~nm}$.

term of Nile red dye concentration $\left(p\right.$ value $\left.=5.129 * 10^{-7}\right)$, and the linear main term of microalgal cell concentration $(p$ value $\left.=3.523 * 10^{-6}\right)$. The main regression coefficients (linear and quadratic) for ultrasonication power and incubation temperature did not show statistical significance over the range investigated. Nile red dye concentration and microalgal cell concentration therefore had more significant effects in changing fluorescence intensity than incubation temperature and ultrasonication power. Among the interaction regression coefficients listed in Table 3, none of them was found to be statistically significant.

Figure 4 compares the values of fluorescence intensity as predicted by the quadratic model (see (4)) against those observed in the experiments. With a determination coefficient $\left(R^{2}\right)$ of 0.86 , the experimental values were found to be well correlated by the quadratic model. Figure 5 shows two out of six response surface plots of the quadratic model (see (4)). In each response plot, two independent variables (in coded values) were simultaneously modified to compute the fluorescence intensity.

3.2.3. Effect of Microalgal Cell Concentration. As previously discussed, fluorescence intensity in the stained growth medium resulted from background dye/medium interaction, while fluorescence intensity in the stained microalgal culture was the combined outcome of dye/lipid interaction and dye/medium interaction. Observed fluorescence intensity for all experiments that stained growth medium (coded value of microalgal cell concentration $=0$, coded value of Nile red dye concentration $=0$ or 1.5) averaged out at $79.86 \pm 29.555 \mathrm{afu}$, while observed fluorescence intensity for experiments that stained microalgal culture (coded value of microalgal cell concentration $=1$, coded value of Nile red dye concentration $=0$ or 1.5) averaged out at a distinctly higher value of $133.73 \pm$ 32.559 afu.

The model obtained a positive value (65.95) for the regression coefficient of microalgal cell concentration (Table 3), thus suggesting the dependence of fluorescence intensity on cell concentration. In a separate experiment where we stained serial dilutions of our microalgal culture (results not shown), we were able to verify the direct dependence of fluorescence intensity on cell concentration of up to $0.11 \mathrm{~g}$ dried microalgae/L feed mixture. These findings agree with previous studies that have reported the linear dependence of Nile red fluorescence with cell concentration [6-8]. This direct dependence was expected as higher cell concentration increased the amount of available lipid per unit volume and led to more frequent dye-lipid interaction.

3.2.4. Effect of Ultrasonication Power. In these experiments, we were using ultrasonication power as a proxy for the level of cell disruption. Disrupting microalgal cells releases intracellular lipid to the surrounding medium, thus allowing the Nile red molecule to bypass membrane permeation and to directly interact with the lipid.

Ultrasonication of the microalgal culture (coded value of microalgal cell concentration $=1$ ) at $0 \mathrm{~W}$ (coded value of ultrasonication power $=-1), 65 \mathrm{~W}$ (coded value of ultrasonication power $=0$ ), and $135 \mathrm{~W}$ (coded value of ultrasonication power $=1$ ) disrupted $0 \%, 38.5 \%$, and $95.0 \%$ of intact cells, respectively. Based on the relatively high $p$ values of its main regression coefficients (Table 3), ultrasonication power was concluded to have little effect on changing fluorescence intensity. This conclusion was verified by the $3 \mathrm{D}$ plot in Figure 5(a), whereby continuous increase in ultrasonication power was not observed to significantly affect the values of the fluorescence intensity.

The lack of correlation between cell rupture and fluorescence intensity suggested that the cell wall/membrane structure of the Tetraselmis strain used for our study was completely permeable to penetration by Nile red dye. Increased cell rupture by ultrasonication released intracellular lipids and improved their accessibility to the dye. This increased access, however, was not needed as our cell wall/membrane structure was completely permeable to the dye and all of the stainable lipids were already successfully stained in the intact cells.

3.2.5. Effect of Incubation Temperature. The relatively high $p$ values obtained for the main regression coefficients of incubation temperature (Table 3 ) indicated that incubation temperature had little influence in changing fluorescence intensity. In Figure 5(b), a continuous increase in incubation temperature was demonstrated to have little effect on the values of fluorescence intensity. This lack of correlation between incubation temperature and fluorescence intensity was unexpected as temperature increase was anticipated to speed up molecular collisions, facilitated more rapid lipid/dye interaction, and increased rate constant. Perhaps the range of temperature change we selected (between 20 and $40^{\circ} \mathrm{C}$ ) was not sufficient to effect any noticeable change in fluorescence intensity.

3.2.6. Effect of Nile Red Dye Concentration. We speculated fluorescence intensity to increase with a rise in the dye concentration. The findings from our statistical analysis, 


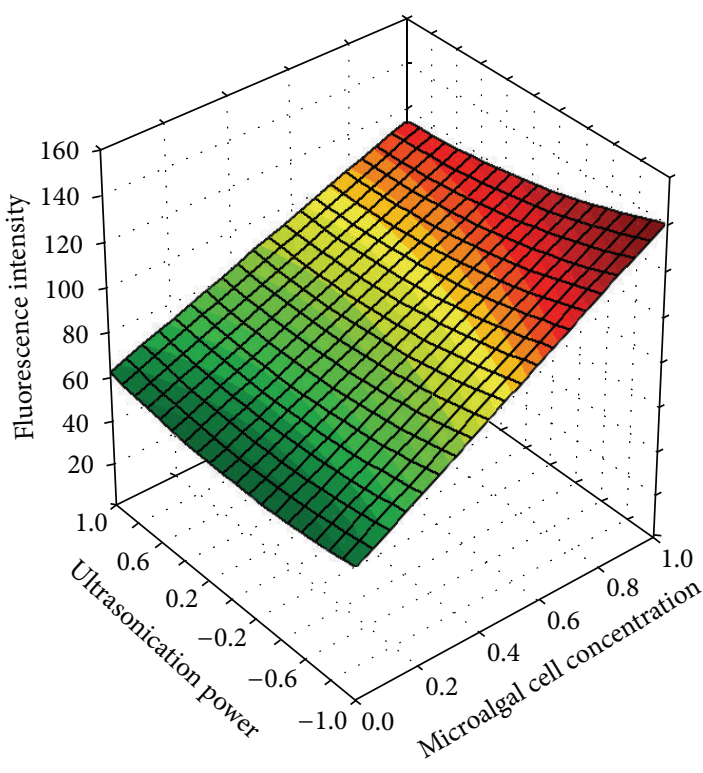

(a)

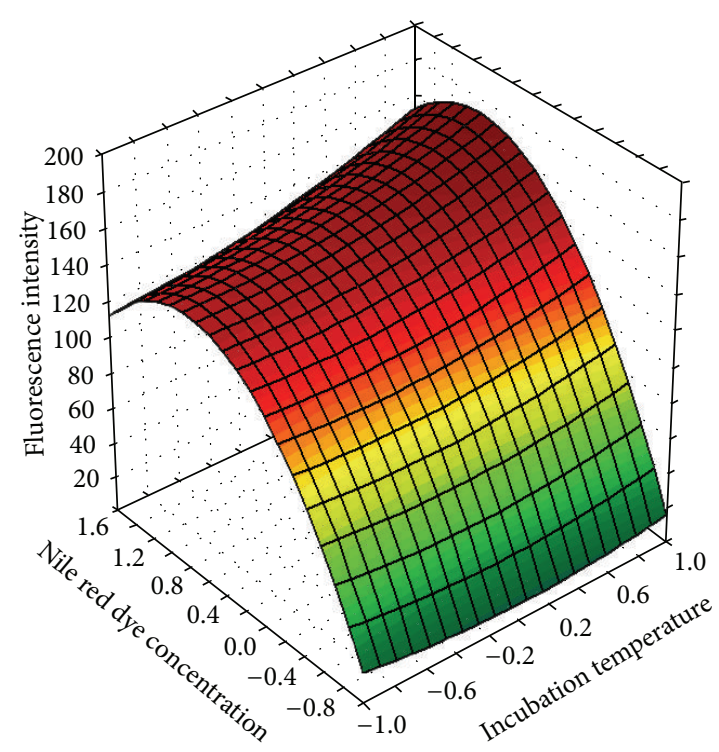

(b)

FIGURE 5: 3D response surface plots of fluorescence intensity as predicted by the quadratic model. In each plot, the effects of two independent variables (in coded values) on fluorescence intensity are simultaneously demonstrated. For each surface plot, the other independent variables which do not form the plot axes are fixed (in coded values): (a) incubation temperature $=0$, Nile red dye concentration $=0$; (b) microalgal cell concentration $=1$, ultrasonication power $=0$. Excitation wavelength $=530 \mathrm{~nm}$, emission wavelength $=607 \mathrm{~nm}$, and poststaining time $=$ $15 \mathrm{~min}$.

however, demonstrated a nonlinear relationship between dye concentration and fluorescence intensity. Because of the negative regression value $(-42.62)$ of the quadratic main effect for Nile red dye concentration (Table 3), fluorescence intensity was found to yield a maxima when plotted as a function of Nile red dye concentration (Figure 5(b)). At a lower dye concentration, fluorescence intensity increased with dye concentration. This was expected as higher dye concentration would increase the frequency of dye/lipid interaction. Beyond the optimal dye concentration level, however, this trend was reversed and the fluorescence value diminished with dye concentration. The quenching of Nile red fluorescence at very high dye concentration has been reported by several studies $[7,9,15]$. Even though its mechanism is not clearly understood, the phenomenon is often attributed to oligomerization (self-stacking) of the dye. At high concentration, the Nile red dye molecules had a somewhat increased tendency to interact with each other. Because of this self-interaction, the dye molecules started stacking on top of each other on the water/lipid interface and became increasingly unavailable for interaction with the lipid globules.

Under our experimental conditions, this optimum dye concentration was found to be $4.69 \mu \mathrm{g}$ dye $/ \mathrm{mL}$ culture (this was equivalent to $42.6 \mu \mathrm{g}$ dye $/ \mathrm{mg}$ dried microalgae or $146.6 \mu \mathrm{g}$ dye $/ \mathrm{mg}$ crude lipid or $3.01 \times 10^{-6} \mu \mathrm{g}$ dye/cell). As can be seen in Table 4, previous studies have reported different values for optimal Nile red concentration. The discrepancy between these values can be attributed to the difference in the lipid contents and composition of the investigated microalgal species. Variation in lipid content and composition directly affects the level of Nile red dye needed for lipid saturation.
We carried out a simple staining study of our strain at logarithmic and stationary growth phase to evaluate if the optimized staining procedure can be applied across different physiological states. As they transition through the growth phases (from logarithmic to stationary), microalgal cells experience various biochemical and physiological changes [4]; the two which appear to be most important for Nile red staining are the increase in the amount of lipid content per unit volume affecting the frequency of lipid/dye interaction and the change in cell wall composition and thickness potentially affecting the ability of the dye to permeate into the cell. We harvested from the photobioreactor at the two different states of the growth phase. To isolate the effect of the change in cell wall/membrane permeability, the samples were concentrated accordingly so that they ended with equal volumetric lipid content after the concentration step. We stained these two samples under several parameters: ultrasonication power at $0 \mathrm{~W}$, incubation temperature at $20-40^{\circ} \mathrm{C}$, and Nile red dye concentration between 2.5 and $6.25 \mathrm{mg}$ dye/L feed mixture. Similar fluorescence levels were obtained for both sets of samples (155.60 \pm 28.68 afu for logarithmic phase, $157.52 \pm 37.94$ afu for stationary phase), suggesting that, within the limit of our experimental conditions, the change in the permeability of cell wall/membrane structure was not statistically significant to affect Nile red staining efficiency. This is consistent with our findings in Section 3.2.4 which seems to suggest the complete Nile red permeability of the cell wall/membrane structure of the Tetraselmis strain used in our study. Nile red staining can thus be used to monitor lipid content of our microalgal strain across different physiological states. 


\subsection{Review of Microalgal Nile Red Staining}

3.3.1. Findings from Previous Studies. Table 4 summarizes the findings from previous studies on microalgal Nile red staining. As shown by the table, there is a significant variability in the range of optimum values found across the studies. Optimum values for the investigated parameters, in particular those of Nile red concentration and staining time, appear to be strongly affected by the microalgal species used in the particular study. This discrepancy is likely to have arisen due to physiological difference in the structure and composition of microalgal cell wall as well as the variation in lipid content and composition between the microalgal species.

Two conclusions can be derived out of the variability observed in Table 4. The first conclusion is that microalgal lipid estimation based on the use of Nile red staining should only be made once the staining method has been optimized for the specific species. Optimization results from a particular species are not universal and should not be directly applied to other species. For example, one species may have an optimum poststaining time of $5 \mathrm{~min}$ while the other has an optimum time of 10 min; a general procedure of measuring fluorescence at $5 \mathrm{~min}$ after staining based on the optimum time of the first species will result in the underestimation of the lipid content of the second species and lead to the erroneous deduction that the first species contains more lipids than the second.

Because of the wide interspecies variability in the optimum staining conditions, the use of Nile red staining on a particular microalgal species needs to be carefully refined to take into account the specific requirements of the species. Using a single staining protocol over a wide range of species without catering to the need of each species will lead to inaccurate estimation of their lipid contents. Once optimized, however, the staining method can be used as a simple diagnostic tool for monitoring lipid quantity of the microalgal species under various environmental and growth conditions.

The second conclusion arising from Table 4 is that there is a critical need for simple operating guidelines that streamline the Nile red optimization process across different microalgal species. By identifying significant variables, the guidelines will help future studies to fine-tune their Nile red staining methods according to the specific needs of their species. This will create a more uniform staining procedure across the different microalgal species, thereby improving the robustness and reliability of the staining method.

\subsubsection{Operating Framework for the Optimization of Microalgal} Nile Red Staining. We thus propose the six-step operating framework as shown in Figure 6 as a general set of guidelines when developing Nile red staining procedure for a particular microalgal species.

Step 1 (verification of the lipid content of the microalgal species). Verify the lipid content of the given culture of the microalgal species. A chloroform/methanol/water solvent mixture at $1: 2: 0.8 \mathrm{v} / \mathrm{v} / \mathrm{v}$ [22] or Soxhlet extraction as described in Section 2.3 can be used for lipid extraction. To fractionate the lipid extract to neutral and polar lipid

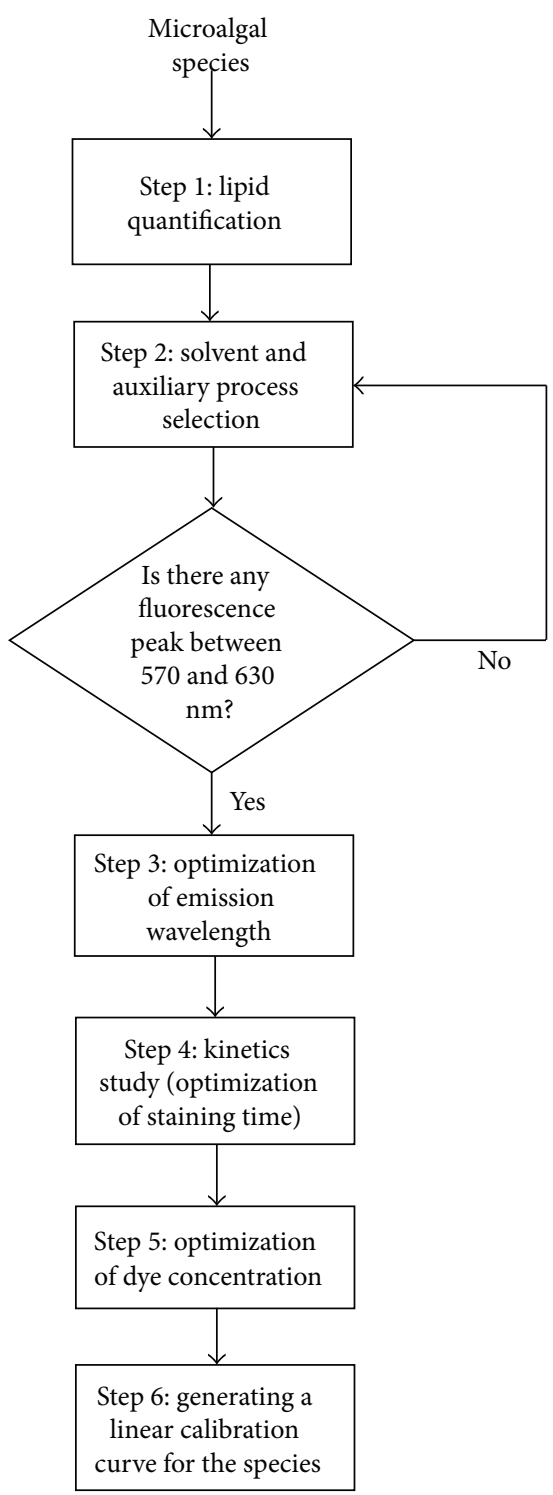

FIGURE 6: Operating framework for the optimization of Nile red staining of a new microalgal species.

fractions, solid phase extraction (SPE) is used. If the lipid content of the culture is less than $3 \mathrm{wt} \%$ of the dry biomass, the species is not lipid-rich and the Nile red staining method is unlikely to be sensitive enough to detect changes in its lipid content. All of the subsequent optimization steps are to be carried out with the batch of microalgal culture used for this step.

Step 2 (selection of carrier solvent and the use of auxiliary step). In order to penetrate into the microalgal cell, Nile red dye needs to be dissolved in a relatively polar solvent that is capable of interacting with the polar components of both the cell wall (cellulose) and the cell membrane (phospholipid) $[11,13]$. The choice of carrier solvent is therefore critical to the staining protocol. As shown in Table 4, acetone and DMSO appear to be the most popular choice for carrier solvents. 
Other cosolvents, such as IPA or glycerol, are often added in tangent to the primary carrier solvent in order to further decrease the dye hydrophobicity and improve dye penetration through the cell membrane [13]. Auxiliary method, such as microwave irradiation, applied as either a pretreatment step or poststaining enhancement, has been previously reported to improve the effectiveness of Nile red staining. Microwave irradiation facilitated more rapid dye penetration by increasing molecular collisions and movement speeds between algal cells and dye molecules [11].

As an initial protocol, we recommend staining the microalgal culture with Nile red stock solution in acetone at $100: 1 \mathrm{v} / \mathrm{v}$ at room temperature $\left(20^{\circ} \mathrm{C}\right)$ for 10 minutes. Use $0.1 \mathrm{~g}$ dried microalgae/L culture as cell concentration. The dye concentration in the stock solution should be $0.25 \mathrm{mg} / \mathrm{mL}$ (this equates to $2.5 \mu \mathrm{g}$ Nile red dye/mL culture). This set of parameters is a rough average of the optimum parameters reported in Table 4. For control, stain blank medium under the same parameters. At fixed excitation wavelength $(490 \mathrm{~nm})$, perform fluorescence scanning of the emission spectrum between 560 and $635 \mathrm{~nm}$ for both the stained culture and the stained blank medium. Similar to Figure 3, microalgal lipid will form a peak between these wavelengths; this peak should be distinctly larger than the background peak obtained from the medium-dye interaction. If the peak of your stained culture is about the same size as the peak of the stained medium, then the dye has not managed to permeate into the cell. In this case, choose a different carrier solvent (DMSO or DMF) or apply the use of cosolvents/auxiliary process to improve dye penetration. We recommend referring to specific studies listed in Table 4 for the range of cosolvent ratios and arrangement of the auxiliary step $[6,11,13,15]$.

Step 3 (optimizing emission wavelengths). The interaction of Nile red dye with a specific lipid class emits a distinct fluorescence that peaks at a particular wavelength [11]. As a general rule, the more polar the lipid class is, the more red-shifted (longer wavelength) its emission peak will be. Polar lipids, the primary constituents of cell and organellar membranes, commonly peak between 590 and $635 \mathrm{~nm}$, while neutral lipids globules peak between 560 and $590 \mathrm{~nm}[8,9]$. It is noted that the division of lipid to neutral and polar is a generalized approach which appears to be the most accurate given our currently limited state of knowledge on microalgal Nile red staining. Microalgal neutral lipid consists of triacylglycerol, diacylglycerol, monoacylglycerol, free fatty acids, various hydrocarbons, sterols, and ketones, while microalgal polar lipid consists of glycolipids and phospholipids [1]. Fluorescence response of each of this lipid subclass to Nile red interaction is yet to be understood and will require further investigation involving multiple standard calibrations.

Even though microalgal lipid constitutes various lipid classes, Nile red stained microalgal cells in general emit a single broad peak between the wavelengths of 570 and $630 \mathrm{~nm}$ (Figure 3). The peaks of individual lipid classes appear to have aggregated to form a single broad peak $[11,15]$. The apex position of this combined peak is, however, dependent on the dominant lipid class, thus leading to the differences in optimum wavelength observed in Table 4. Microalgal species with a high proportion of neutral lipid will have a peak apex within the yellow colour range (560-590 nm) while those with a significant proportion of polar lipid will have a peak apex within the red colour range (590-635 nm). In our study, our polar lipid seems to predominate, red-shifting our peak apex to around $607 \mathrm{~nm}$. Because lipid composition varies significantly across different microalgal species, the position of the peak apex and thus the optimal emission wavelength cannot be generalized from one species to another. Examine the emission spectrums of the stained microalgal species and determine the exact position of its peak apex before progressing further with the optimization procedure.

Step 4 (kinetic study (optimizing staining time)). As discussed in Section 3.1, the evolution of Nile red fluorescence in microalgal cells as a function of staining time is generally characterized by rapid enhancement followed by steady quenching (Figure 2) [15]. The reason for fluorescence quenching with extensive incubation time is not clearly understood. In a couple of studies [11, 15], however, the fluorescence quenching over time has been attributed to enzymatic degradation of the dye as part of cellular acclimation to the presence of the exogenous stain. For this reason, it is critical that Nile red fluorescence is evaluated at its optimal poststaining time.

The kinetic study should be carried out with the carrier solvent and at the optimal emission wavelength obtained from previous steps. The fluorescence intensity is to be recorded at regular intervals for $30 \mathrm{~min}$ after staining. Since most of the species in Table 4 seems to reach their optimal time within $15 \mathrm{~min}$ of staining, we recommend frequent measurements within this period (perhaps one reading every minute). Beyond $15 \mathrm{~min}$, the measurement frequency can be lowered to one reading every three minutes.

Step 5 (optimizing dye concentration). As discussed in Section 3.2.5, the evolution of Nile red fluorescence in microalgal cells as a function of dye concentration is also characterized by enhancement followed by quenching (Figure 5) $[7,15,16]$. Quenching at high dye concentration is generally attributed to self-stacking of the dye molecules on the surface of the lipid globules [15].

Refinement of the dye concentration should be carried out at the optimal emission wavelength and staining time obtained from the previous steps. Given the wide range of optimal dye concentration reported in Table 4 (between 0.25 and $10 \mu \mathrm{g} / \mathrm{mL}$ ), we recommend a six-point experimental design whereby the amount of Nile red added to the culture is steadily incremented to yield the following final concentrations: $0.25,0.5,1,2.5,5$, and $10 \mu \mathrm{g} / \mathrm{mL}$.

Step 6 (generating calibration curve). A linear calibration curve that correlates the volumetric lipid content of the microalgal species ( $y$ g lipid/L culture) with its fluorescence intensity at the optimal staining conditions can now be generated. The curve will enable the direct conversion of Nile red staining results to volumetric lipid content. Using the curve, Nile red staining can be applied as a rapid diagnostic tool for evaluating lipid accumulation of the species at 
different stages of its life cycle under various environmental and growth conditions.

From Steps 1 and 2, we have verified both the cell concentration ( $0.1 \mathrm{~g}$ dried microalgae/L culture) and lipid content ( $x \mathrm{~g}$ lipid/g dried microalgae) of the microalgal culture. Record the optical density (OD) of the culture at $\lambda$ $=750 \mathrm{~nm}$ using a spectrophotometer. OD value corresponds directly to the amount of suspended cells, allowing its use as a proxy indicator for cell concentration. Using either serial dilution or concentration by centrifugation produces five different concentrations of the microalgal culture: 0.025 , $0.05,0.1,0.2$, and $0.4 \mathrm{~g}$ dried microalgae/L culture. Simple mathematics should enable calculation of the volumetric lipid content of each diluted or concentrated solution $(y \mathrm{~g}$ lipid/L culture). Stain each of the solutions with the optimized Nile red method. Correlate the resulting fluorescence at optimal emission wavelength with volumetric lipid content to generate the calibration curve.

The calibration curve is now ready to be used for lipid quantification of different cultures of the microalgal species. To find the volumetric lipid content of a particular culture, check the OD of the culture and dilute appropriately until it reaches the OD equivalent to cell concentration of $0.1 \mathrm{~g}$ dried microalgae/L culture. Stain the diluted culture using the optimized staining protocol and compare its fluorescence value to the calibration curve.

3.4. Proposed Mechanism of Nile Red Staining. Nile red staining in microalgal cells is a complex phenomenon, the exact mechanism of which is currently still unknown. Based on the findings from this study as well as other previous studies on microalgal Nile red staining, we are able to piece together a possible mechanism for the staining process. We propose the Nile red fluorescence in microalgal cells to be a five-stage process (Figure 7).

Stage 1 (dissolution of Nile red dye in the carrier solvent). Since Nile red dye is hydrophobic in nature, it needs to be dissolved in a polar carrier solvent to facilitate cell permeation [11, 13]. Please refer to Table 4 for the list of solvents that previous studies have used and Step 2 in Section 3.3.2 for understanding the factors that need to be considered when selecting a carrier solvent.

Stage 2 (diffusion of dye-solvent complex into the cell). The carrier solvent will interact with the polar components of both the cell wall and the cell membrane. This interaction will enable the dye-solvent complex to diffuse across the cell wall/membrane structure and penetrate into the cell.

Stage 3 (transfer of Nile red dye to the lipid globule). The dyesolvent complex will now diffuse within the cell to find the lipid globule. For simplification, Figure 6 has represented all intracellular lipids as a lipid globule (neutral lipid). We note that the Nile red dye can also interact with polar lipid. The fluorescence that will arise from interaction with polar lipid is, however, of a longer wavelength than that generated from neutral lipid interaction (please refer to Section 3.3.2, Step 3) $[14,15]$.

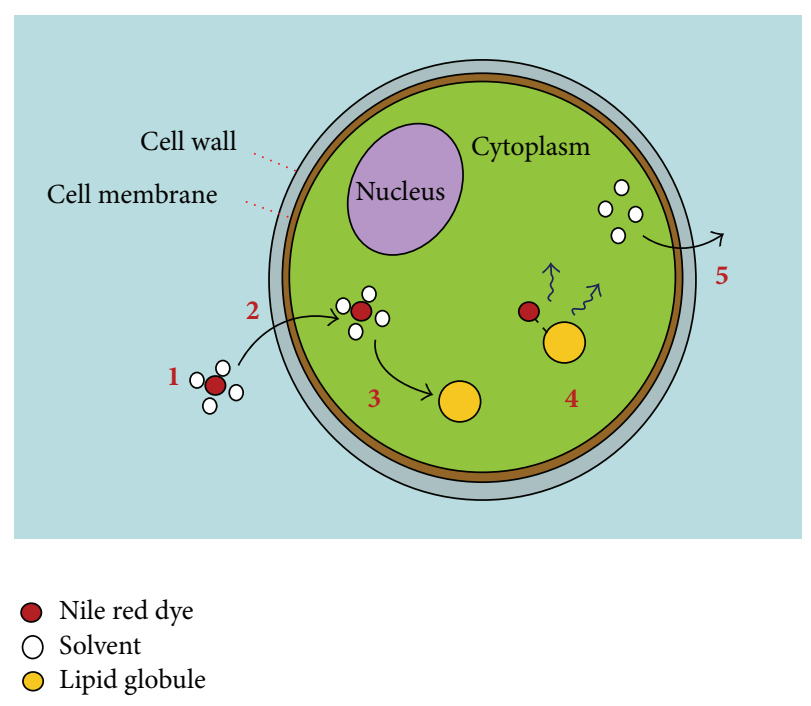

FIGURE 7: A schematic diagram showing the pathway that drives Nile red interaction with microalgal intracellular lipid. Stage 1: dissolution of Nile red dye in the carrier solvent; Stage 2: diffusion of the dye-solvent complex across the cell wall and the cell membrane into the cell; Stage 3: transfer of the dye to the lipid globule; Stage 4: interaction of the dye with the lipid globule resulting in fluorescence; and Stage 5: diffusion of the carrier solvent out of the cell.

Stage 4 (interaction of the Nile red dye with the lipid globule). The dye will bind with the lipid globule to form a dye-lipid complex. As a result of this interaction, the complex is able to absorb blue-green photons (490-530 $\mathrm{nm})$, reach an excited state, and emit photons of lower energy level between 560 and $635 \mathrm{~nm}$ (yellow and red). The stronger the interaction between the dye and the lipid is, the more intense the resulting fluorescence becomes. The strength of dye/lipid interaction is a direct function of the amount of lipid, the ratio of Nile red dye to lipid (or Nile red dye concentration), and the poststaining time $[7,8,11,15]$. Please refer to Section 3.3.2 for the factors that need to be taken into consideration when selecting optimal Nile red dye concentration and poststaining time.

Stage 5 (diffusion of the carrier solvent out of the cell). Residual carrier solvent will now diffuse out of the cell back to the surrounding medium.

\section{Conclusions}

In the near future, microalgal biofuel is predicted to play a significant role in the global provision of sustainable energy. A critical understanding of Nile red behaviour as a rapid diagnostic tool for microalgal lipid quantification is needed for the identification of lipid-rich species and the monitoring of lipid content when optimizing growth conditions.

Through statistical modelling, this study provided new insights into the behaviour of microalgal Nile red staining. A quadratic model that described Nile red fluorescence intensity in microalgal cells (Tetraselmis suecica) as a composite function of several staining variables (microalgal cell 
concentration, ultrasonication power, incubation temperature, and Nile red dye concentration) was developed. From the model, Nile red dye concentration and microalgal cell concentration (as a proxy of lipid content per unit volume) were shown to play critical roles in affecting fluorescence intensity. On the other hand, incubation temperature and ultrasonication power were both found to be statistically insignificant. Fluorescence values predicted by the quadratic model were found to be well correlated with the experimental results $\left(r^{2}=0.86\right)$.

By compiling findings from previous microalgal Nile red research, the study also demonstrated the wide diversity in the staining conditions used across the different species. Using insights gained from this study and previous Nile red studies, we established a simple six-step operating framework for the optimization of Nile red staining procedure across different microalgal strains. The framework enables future studies to accurately and rapidly optimize the staining protocol according to the specific requirements of the species being investigated. This will in turn improve the robustness and reliability of the staining method as a diagnostic tool to measure the amount of accumulated microalgal lipid.

\section{Conflict of Interests}

The authors declare that there is no conflict of interests regarding the publication of this paper.

\section{References}

[1] R. Halim, M. K. Danquah, and P. A. Webley, "Extraction of oil from microalgae for biodiesel production: a review," Biotechnology Advances, vol. 30, no. 3, pp. 709-732, 2012.

[2] R. Halim, T. W. T. Rupasinghe, D. L. Tull, and P. A. Webley, "Modelling the kinetics of lipid extraction from wet microalgal concentrate: a novel perspective on a classical process," Chemical Engineering Journal, vol. 242, pp. 234-253, 2014.

[3] P. M. Schenk, S. R. Thomas-Hall, E. Stephens et al., "Second generation biofuels: high-efficiency microalgae for biodiesel production," BioEnergy Research, vol. 1, no. 1, pp. 20-43, 2008.

[4] A. Widjaja, C.-C. Chien, and Y.-H. Ju, "Study of increasing lipid production from fresh water microalgae Chlorella vulgaris," Journal of the Taiwan Institute of Chemical Engineers, vol. 40, no. 1, pp. 13-20, 2009.

[5] R. H. Wijffels and M. J. Barbosa, "An outlook on microalgal biofuels," Science, vol. 329, no. 5993, pp. 796-799, 2010.

[6] E. Bertozzini, L. Galluzzi, A. Penna, and M. Magnani, "Application of the standard addition method for the absolute quantification of neutral lipids in microalgae using Nile red," Journal of Microbiological Methods, vol. 87, no. 1, pp. 17-23, 2011.

[7] W. Chen, C. Zhang, L. Song, M. Sommerfeld, and Q. Hu, "A high throughput Nile red method for quantitative measurement of neutral lipids in microalgae," Journal of Microbiological Methods, vol. 77, no. 1, pp. 41-47, 2009.

[8] S. J. Lee, B.-D. Yoon, and H.-M. Oh, "Rapid method for the determination of lipid from the green alga Botryococcus braunii," Biotechnology Techniques, vol. 12, no. 7, pp. 553-556, 1998.

[9] G.-H. Huang, G. Chen, and F. Chen, "Rapid screening method for lipid production in alga based on Nile red fluorescence," Biomass and Bioenergy, vol. 33, no. 10, pp. 1386-1392, 2009.
[10] K. Kimura, M. Yamaoka, and Y. Kamisaka, "Rapid estimation of lipids in oleaginous fungi and yeasts using Nile red fluorescence," Journal of Microbiological Methods, vol. 56, no. 3, pp. 331338, 2004.

[11] W. Chen, M. Sommerfeld, and Q. Hu, "Microwave-assisted Nile red method for in-vivo quantification of neutral lipids in microalgae," Bioresource Technology, vol. 102, no. 1, pp. 135-141, 2011.

[12] K. E. Cooksey, J. B. Guckert, S. A. Williams, and P. R. Callis, "Fluorometric determination of the neutral lipid content of microalgal cells using Nile Red," Journal of Microbiological Methods, vol. 6, no. 6, pp. 333-345, 1987.

[13] T.-T. Y. Doan and J. P. Obbard, "Improved Nile Red staining of Nannochloropsis sp.", Journal of Applied Phycology, vol. 23, no. 5, pp. 895-901, 2011.

[14] D. Elsey, D. Jameson, B. Raleigh, and M. J. Cooney, "Note: fluorescent measurement of microalgal neutral lipids," Journal of Microbiological Methods, vol. 68, no. 3, pp. 639-642, 2007.

[15] U. Pick and T. Rachutin-Zalogin, "Kinetic anomalies in the interactions of Nile red with microalgae," Journal of Microbiological Methods, vol. 88, no. 2, pp. 189-196, 2012.

[16] J. T. Cirulis, B. C. Strasser, J. A. Scott, and G. M. Ross, “Optimization of staining conditions for microalgae with three lipophilic dyes to reduce precipitation and fluorescence variability," Cytometry Part A, vol. 81, no. 7, pp. 618-626, 2012.

[17] C. Cubas, M. G. Lobo, and M. González, "Optimization of the extraction of chlorophylls in green beans (Phaseolus vulgaris L.) by $N, N$-dimethylformamide using response surface methodology," Journal of Food Composition and Analysis, vol. 21, no. 2, pp. 125-133, 2008.

[18] P. Thana, S. Machmudah, M. Goto, M. Sasaki, P. Pavasant, and A. Shotipruk, "Response surface methodology to supercritical carbon dioxide extraction of astaxanthin from Haematococcus pluvialis," Bioresource Technology, vol. 99, no. 8, pp. 3110-3115, 2008.

[19] M. G. Sajilata, R. S. Singhal, and M. Y. Kamat, "Supercritical $\mathrm{CO}_{2}$ extraction of $\gamma$-linolenic acid (GLA) from Spirulina platensis ARM 740 using response surface methodology," Journal of Food Engineering, vol. 84, no. 2, pp. 321-326, 2008.

[20] Y. Chisti, "Biodiesel from microalgae," Biotechnology Advances, vol. 25, no. 3, pp. 294-306, 2007.

[21] L. Wang and C. L. Weller, "Recent advances in extraction of nutraceuticals from plants," Trends in Food Science and Technology, vol. 17, no. 6, pp. 300-312, 2006.

[22] I. L. D. Olmstead, D. R. A. Hill, D. A. Dias et al., "A quantitative analysis of microalgal lipids for optimization of biodiesel and omega-3 production," Biotechnology and Bioengineering, vol. 110, no. 8, pp. 2096-2104, 2013. 

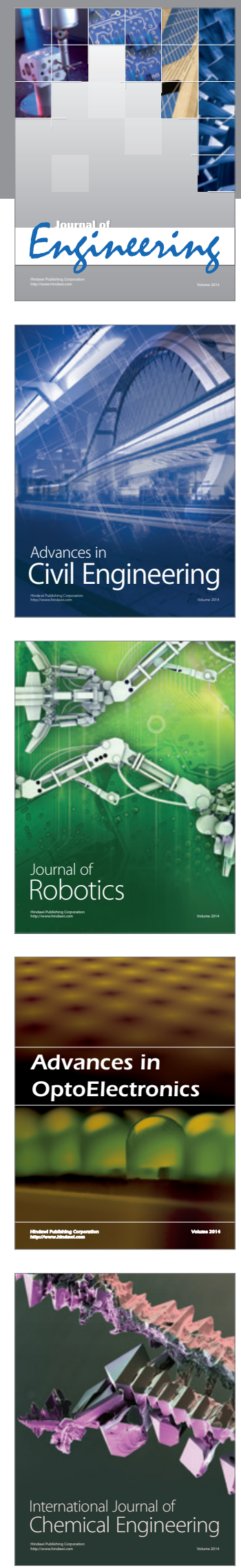

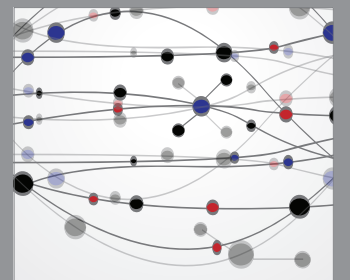

The Scientific World Journal
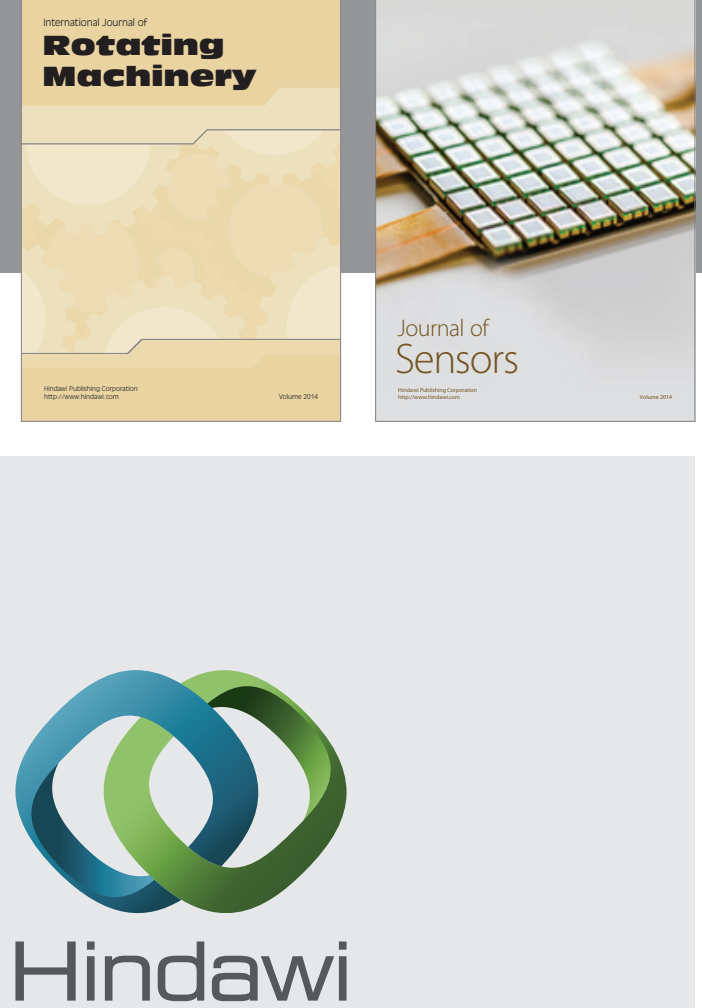

Submit your manuscripts at http://www.hindawi.com
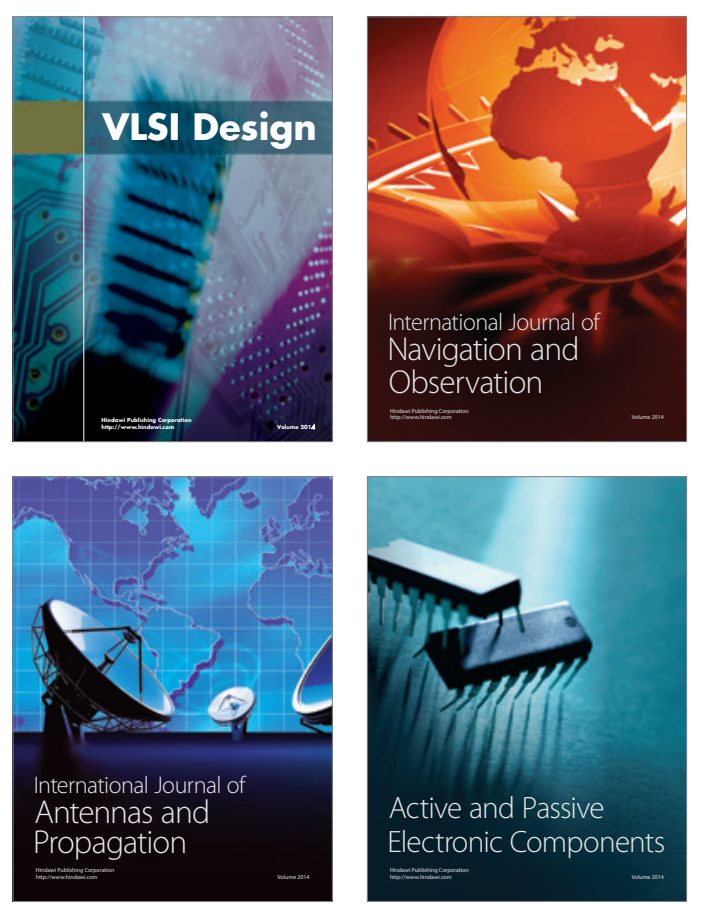
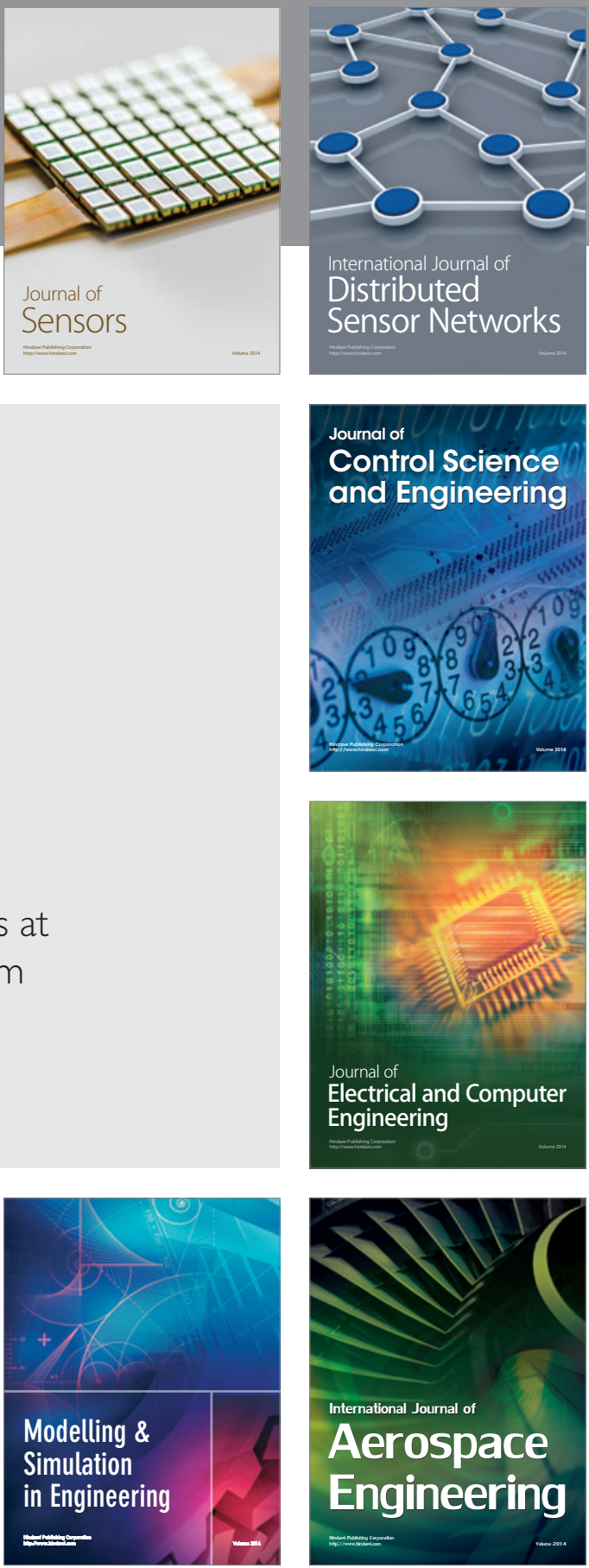

Journal of

Control Science

and Engineering
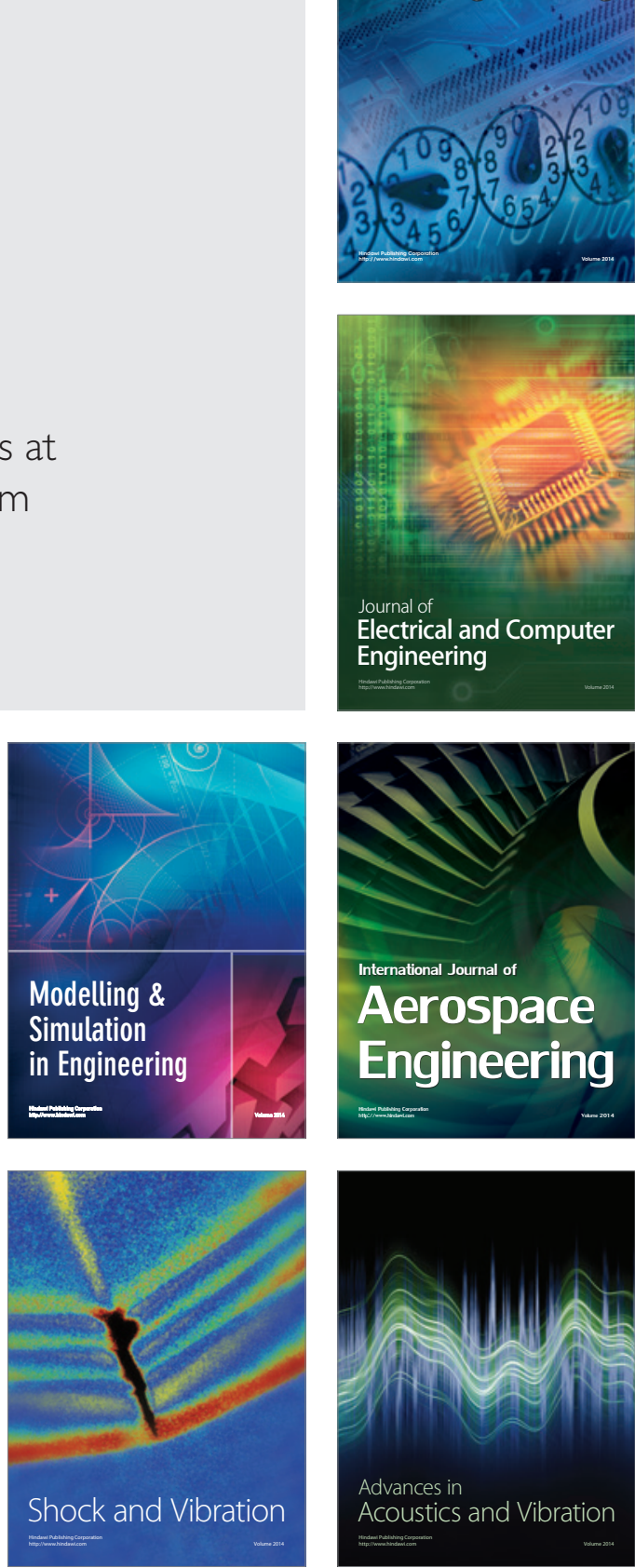